\title{
Application of a Flush Airdata Sensing System to a Wing Leading Edge (LE-FADS)
}

Stephen A. Whitmore, Timothy R. Moes, Mark W. Czerniejewski, and Douglas A. Nichols

Dryden Flight Research Facility

Edwards, California

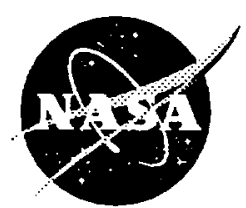

National Aeronautics and

Space Administration

Dryden Flight Research Facility

Edwards, California 93523-0273 
- 


\title{
APPLICATION OF A FLUSH AIRDATA SENSING SYSTEM TO A WING LEADING EDGE (LE-FADS)
}

\author{
Stephen A. Whitmore* \\ Timothy R. Moes* \\ Mark W. Czerniejewski** \\ Douglas A. Nichols** \\ NASA Dryden Flight Research Facility \\ P.O. Box 273 \\ Edwards, California 93523-0273
}

\begin{abstract}
This paper investigates the feasibility of locating a flush airdata sensing (FADS) system on a wing leading edge where the operation of the avionics or fire control radar system will not be hindered. The leadingedge FADS system (LE-FADS) was installed on an unswept symmetrical airfoil and a series of low-speed wind-tunnel tests were conducted to evaluate the performance of the system. As a result of the tests it is concluded that the aerodynamic models formulated for use on aircraft nosetips are directly applicable to wing leading edges and that the calibration process is similar. Furthermore, the agreement between the airdata calculations for angle of attack and total pressure from the LE-FADS and known wind-tunnel values suggest that wing-based flush airdata systems can be calibrated to a high degree of accuracy. Static wind-tunnel tests for angles of attack from $-50^{\circ}$ to $50^{\circ}$ and dynamic pressures from 3.6 to $11.4 \mathrm{lb} / \mathrm{ft}^{2}$ were performed.
\end{abstract}

\section{Nomenclature}

$\begin{array}{ll}A & \text { general aerodynamic coefficient } \\ B & \text { general aerodynamic coefficient } \\ C m_{L E} & \begin{array}{c}\text { pitching moment at the wing leading } \\ \text { edge }\end{array} \\ & \text { pressure coefficient } \\ C_{p} & \text { lower surface pressure coefficient } \\ C_{p_{\ell}} & \text { upper surface pressure coefficient } \\ C_{p_{u}} & \text { wing chord, in. } \\ c & \text { sectional lift coefficient } \\ c_{\ell} & \end{array}$

\footnotetext{
Aerospace Engineer. Member AIAA.

* Engineering student trainee.

Copyright (C) 1993 by the American Institute of Aeronautics and Astronautics, Inc. No copyright is asserted in the United States under Title 17, U.S. Code. The U.S. Government has a royalty-free license to exercise all rights under the copyright claimed herein for Governmental purposes. All other rights are reserved by the copyright owner.
}

ESP

FADS electronically scanned pressure

HARV

HI-FADS high-angle-of-attack flush airdata sensing system

LE-FADS leading-edge flush airdata sensing system

$L^{\prime} \quad$ sectional lift, $\mathrm{lbm} / \mathrm{ft}$

$M_{\infty} \quad$ Mach number

$P_{0} \quad$ tunnel total (ambient) pressure, $\mathrm{lb} / \mathrm{ft}^{2}$

$P_{\infty} \quad$ tunnel free-stream static pressure, $\mathrm{lb} / \mathrm{ft}^{2}$

$q_{\max }$ dynamic pressure at maximum tunnel $\mathrm{r} / \mathrm{min}$

$q_{\infty}$

$R$

RT-FADS

$V_{\infty}$

$x$

$x / c$

$x_{c p}$

$\Gamma$

$\alpha$

$\alpha_{e f f}$

$\delta \alpha$

$E$

$\lambda$

$\theta$

$\rho$ free-stream incompressible dynamic pressure, $\mathrm{lb} / \mathrm{ft}^{2}$

vortex radius, in.

real-time flush airdata sensing system

tunnel airspeed, kn

longitudinal coordinate, $\mathrm{ft}$

normalized longitudinal coordinate

wing center of pressure

vortex circulation strength, $\mathrm{ft}^{2} / \mathrm{sec}$

geometric angle of attack, deg on $C_{p}$, deg

difference between effective and geometric angle of attack, deg

FADS aerodynamic calibration coefficient port latitude angle, deg

wind vector incidence angle to surface, deg

density, $\mathrm{lbm} / \mathrm{ft}^{2}$ effective angle of attack calculated based 


\section{Introduction}

Airmass reference data for flight vehicles-traditionally referred to as airdata-always have been critical measurement parameters for the flight test community. Historically, airdata measurements were performed using intrusive booms which extend beyond the local flow field of the aircraft and measure airmass velocities by direct stagnation of the flow via a pitot tube at the end of the boom. Flow incidence angles were measured using mechanical vanes attached to the probe. Localized aircraft-induced effects were removed through empirical calibration. The National Advisory Committee for Aeronautics (NACA) standardized the design and calibration of these measurement booms. ${ }^{1}$ While the booms performed well at making steady measurements at low-to-moderate angles of attack, the booms were sensitive to vibration and alignment error, and susceptible to damage. Furthermore, specialized requirements of advanced vehicles such as the space shuttle, the National Aero-Space Plane (NASP), and the B-2 Stealth Bomber, make the use of conventional intrusive airdata measurement systems highly undesirable.

As a means of circumventing these and other difficulties with intrusive systems, the flush airdata sensing (FADS) system concept-where airdata are inferred from nonintrusive surface pressure measurementswas developed at the NASA Langley Research Center (NASA-Langley) for the space shuttle program. The FADS technique was adapted to aeronautical applications at the NASA Dryden Flight Research Facility (NASA-Dryden), where several FADS demonstration programs have been performed.

The original program, the shuttle entry airdata sensing (SEADS) system, was developed for the space shuttle and demonstrated the feasibility of the concept. ${ }^{2}$ Following the SEADS program, early aeronautical applications included programs conducted on the $\mathrm{KC}$ 135 and F-14 vehicles. ${ }^{3,4}$ Early FADS analyses used only selected ports, chosen empirically, as inputs to arbitrary curve-fitting schemes which related measured pressure differences to airdata parameters. The emphasis of these flight programs was on measurement and presentation of individual pressure coefficient data and their specific empirical relationships to airdata.

A more advanced program, flight-tested at NASADryden, developed a flush measurement system capable of operating at high angles of attack. The resulting system, the high-angle-of-attack flush airdata sensing (HI-FADS) system, recently concluded flighttesting during phase one of the high alpha program conducted on the F-18 High Alpha Research Vehicle (HARV) at NASA-Dryden. ${ }^{5}$ The system, used primarily for research measurements, is an evolution of the earlier nonintrusive systems and emphasized the entire airdata system development, including aerodynamic modeling, algorithms, and system redundancy. The algorithms developed during the HARV flight tests were coded to be real-time capable. In addition, a considerable development effort was made to ensure algorithm robustness and fault tolerance. The faultmanagement development ensures that the system can run autonomously without ground-based intervention. Excellent results were achieved for flight conditions up to $50^{\circ}$ angle of attack $(\alpha)$ and 1.20 Mach number. Results of these flight tests have been reported previously in Refs. 5,6 , and 7 . An overview of failuredetection and fault-management techniques developed for the real-time (RT)-FADS system are presented in Ref. 8.

Low-speed wind-tunnel tests also were performed using the HI-FADS system installed on a full-scale F-18 forebody. These tests, performed in the NASA-Langley 30 - by $60-\mathrm{ft}$ wind tunnel, at a maximum dynamic pressure of $12.4 \mathrm{lb} / \mathrm{ft}^{2}$, gave results which were remarkably similar to results achieved during the HARV flight tests. From these tests it was concluded that flight and wind-tunnel test results for FADS systems are well correlated. A brief summary of the preliminary windtunnel test results and comparisons to flight data are presented in Ref. 9.

All of the previously mentioned systems utilize a matrix of ports in which pressures are sensed in the vicinity of the fuselage nosetip. The nosetip was chosen as a primary location for the FADS systems for several reasons: (1) the effect of aircraft-induced upwash was considered to be minimal in this location, (2) it was believed that the nosetip region would remain unseparated throughout a large angle-of-attack envelope, and (3) most importantly, the nosetip has been used traditionally as the airdata measurement location. Unfortunately, installation of FADS sensors and the associated electronics at the nosetip complicates the design and operation of the aircraft radar, a critical feature of most high-performance aircraft.

This paper investigates the feasibility of locating a FADS system on a wing leading edge (LE-FADS) where the operation of the avionics or fire control radar system will not be hindered. Tests to be described in this paper will verify that the aerodynamic model developed for nosetip locations is directly applicable to a wing leading edge. The feasibility of a wing-based FADS system will be demonstrated using data derived from a low-speed wind-tunnel test of an unswept symmetrical airfoil. Wing aerodynamic characteristics, measurement matrix configuration, instrumentation, and test techniques will be described. All results presented in this report are static data. 
It is intended that later tests will be performed using swept-wing configurations, nonsymmetrical airfoils, sharper leading edges, and dynamic conditions. Later algorithm formulations will include the full airdata state in which angle of sideslip also will be estimated.

\section{FADS Wing Test Section}

In order to demonstrate the feasibility of an LEFADS system, a simple wing configuration was constructed, and a pressure matrix was installed on the leading edge. The symmetric airfoil used in the tunnel tests is shown in Fig. 1. The leading edge had a circular radius of 0.25 in. The wing had a 4.2 -in. chord and a maximum thickness-to-chord ratio of 0.119 . A 3.5-in. span allowed 0.25 in. of lateral clearance at the walls to mitigate tunnel boundary-layer effects.

The aerodynamic characteristics of the airfoil were analyzed using the Program for ANalysis and Design of Airfoils (PANDA). ${ }^{10}$ Pressure coefficient distributions resulting from the analysis are presented in Fig. 2(a) at $\alpha=0^{\circ}$, and in Fig. 2(b) at $\alpha=10^{\circ}$. The sectional lift coefficient $\left(c_{\ell}\right)$ of the wing was evaluated over an angle-of-attack range from $-50^{\circ}$ to $50^{\circ}$. These data are presented in Fig. 2(c). Since the net moment at the wing center of the pressure is zero, ${ }^{11}$

$$
C m_{L E}+c_{\ell} x_{c P}=0
$$

the wing center of pressure was evaluated by integrating the leading-edge moment induced by the upper and lower pressure distributions along the length of the airfoil and dividing by the sectional lift coefficient.

$$
x_{c p}=\frac{\int_{a}^{1} \frac{x}{c}\left[C_{\left.\left.p_{\ell}\left(\frac{x}{c}\right)-C_{p_{u}\left(\frac{x}{c}\right)}\right)\right] d\left(\frac{x}{c}\right)}\right.}{c_{\ell}}
$$

Over the range from $\alpha=-50^{\circ}$ to $50^{\circ}$, the center of pressure was found to vary from 0.2275 to 0.2284 percent of the chord, with a mean value of 0.2280 percent. The steep pressure gradients near the leading edge, and the strong variation of the upper and lower pressure distributions as a function of angle of attack, offer a wealth of information from which the airdata values may be estimated.

\section{Instrumentation System and Wind-Tunnel Test Section}

The FADS wing section was tested in a low-speed wind tunnel at the NASA-Dryden Fluids Lab Test Facility (FLTF). The tunnel has a 4 - by 10 -in. test section approximately $18 \mathrm{in}$. long. The tunnel has a top airspeed of approximately $70 \mathrm{kn}$, and (at the $2200 \mathrm{ft}$ elevation of the facility) a maximum dynamic pressure of approximately $12 \mathrm{lb} / \mathrm{ft}^{2}$. Tunnel flow is forced by a "squirrel cage" fan, powered by a variable-speed threephase motor. A plenum at the upstream end of the tunnel uses "honeycomb" flow straighteners to minimize inlet flow distortion. A schematic of the test measurement system is depicted in Fig. 3.

The airfoil was instrumented with nine pressure ports placed at $20^{\circ}$ increments along the leading edge. Measured relative to the axis of symmetry, the latitude angles $(\lambda)$ of these ports were at $\pm 80^{\circ}, \pm 60^{\circ}, \pm 40^{\circ}$, $\pm 20^{\circ}$, and $0^{\circ}$. The ports were staggered along a $45^{\circ}$ incline with the rotational axis to prevent flow interference with neighboring ports. The wing was mounted in the tunnel so that the pivot point was at 0.5 normalized longitudinal coordinate $(x / c)$. Steel pressure tubing of 0.025 inner diameter and 0.04 outer diameter was passed through the pivot point of the airfoil and routed to the pressure ports. Geometric angle of attack was measured by using an indicator fastened to the rotating axis of the airfoil and a protractor located on top of the tunnel.

The FADS pressure measurements were obtained with an electronically scanned pressure (ESP) module with 16 individual pressure transducers packaged in a single module with a single analog output. Wing pressures were transported to the ESP module using lengths of flexible pneumatic tubing. The ESP module measured differential pressure, with respect to room pressure, with an accuracy of $\pm 0.1 \mathrm{lb} / \mathrm{ft}^{2}$. The ESP module was fastened to the side of the wind tunnel at an elevation corresponding to that of the leading-edge ports. This was done to minimize error of the reference static pressure port located on the ESP module. The wing pressure values were sampled by using ESP module ports one through nine. Tunnel static pressure was collected by connecting several tunnel test section static ports together using a pressure manifold. Sampling was done by using the tenth ESP module pressure port. Room ambient pressure was measured using a high-accuracy analog barometer.

The zero-level readings of the individual transducers of the ESP module were found to vary significantly as a function of time, with a maximum variation of $-0.5 \mathrm{lb} / \mathrm{ft}^{2} / \mathrm{hr}$. Since these shifts were a considerable portion of the overall pressure readings, an automated procedure was developed, in which the zero readings for each transducer were measured and subtracted from the pressure reading prior to each test run. Since the duration of each test point was approximately $60 \mathrm{sec}$, the drift inaccuracy of the adjusted ESP module pressure measurement $\left(0.05 \mathrm{lb} / \mathrm{ft}^{2}\right)$ over the course of the test is estimated to be smaller than the absolute transducer error. 


\section{Test Procedure}

The ESP module pressure data were sampled using a 16-bit analog-to-digital conversion board installed in a microcomputer. Individual pressure transducers were selected from the ESP module using a 4-bit parallel digital address output from the microcomputer. For test validation, the ESP module pressure data were also displayed in real time during the tests. For each test, data were collected for approximately $60 \mathrm{sec}$, with a full data frame (all nine pressure ports and tunnel static pressure) collected approximately 5 times a sec. At the end of each test all of the data were time-averaged to reduce the effects of random measurement noise.

Tests were run for angles of attack ranging from $-50^{\circ}$ to $50^{\circ}$ at three separate tunnel speed conditions: $35 \mathrm{kn}$, $45 \mathrm{kn}$, and at a maximum fan shaft setting of $1750 \mathrm{rev}$ olutions per minute ( $r / \mathrm{min}$ ), which, depending on angle of attack and tunnel blockage, yielded an airspeed that varied from 50 to $65 \mathrm{kn}$. Figure 4(a) presents the percentage of tunnel blockage as a function of the wing angle of attack. The main effect of the tunnel blockage was to limit the airspeed obtained for a given fan shaft $\mathrm{r} / \mathrm{min}$ setting. To maintain constant tunnel speed, the fan shaft $\mathrm{r} / \mathrm{min}$ setting was varied to compensate for tunnel blockage. Figure $4(\mathrm{~b})$ shows the maximum obtainable velocity as a function of angle of attack (at $1750 \mathrm{r} / \mathrm{min}$ ). As can be seen from the figure, 32.2-percent blockage at $\alpha=50^{\circ}$ corresponded to a 22.7-percent reduction in tunnel velocity. Dynamic pressure for these tests ranged from 3.6 to $11.4 \mathrm{lb} / \mathrm{ft}^{2}$. Figure 4(c) shows the maximum obtainable dynamic pressure (at a shaft speed of $1750 \mathrm{r} / \mathrm{min}$ ) as a function of angle of attack. For each tunnel speed setting, the initial test run was conducted at $\alpha=0^{\circ}$, after which succeeding tests were cycled to $\alpha=50^{\circ}$ at $5^{\circ}$ increments. After the $\alpha=50^{\circ}$ test point, secondary tests at $\alpha=10^{\circ}$ and $30^{\circ}$ were initiated to verify repeatability. A similar approach was undertaken for the tests run from $\alpha=0^{\circ}$ to $-50^{\circ}$.

\section{Modeling and Analysis}

The aerodynamic model, which relates the measured pressure data to the desired airdata quantities, is developed in a manner analogous to the model development presented in Ref. 5. For these tests, three airdata parameters, dynamic pressure $\left(q_{\infty}\right)$, angle of attack $(\alpha)$, and static pressure $\left(p_{\infty}\right)$, were included in the model. Since the test section is two-dimensional, angle of sideslip was zero for all of these tests. For incompressible potential flow around an infinite twodimensional cylinder, the pressure coefficient at the surface is given by

$$
C_{p}(\theta)=1-4 \sin ^{2}(\theta)=-3+4 \cos ^{2}(\theta),
$$

where $\theta$ is the flow incidence angle between the wind velocity vector and the normal to the surface at the port. To account for compression and tunnel blockage effects, the coefficients are allowed to assume arbitrary values while still retaining the basic form of the model, i.e.,

$$
C_{p}(\theta)=A+B \cos ^{2}(\theta)
$$

In order to satisfy conservation of momentum, the stagnation pressure constraint must be enforced (Ref. 5), i.e., when $\theta=0$,

$$
C_{p}(\theta=0)=A+B=1
$$

This constraint may be built into the model by letting

$$
A=\varepsilon, \text { and } B=(1-\varepsilon),
$$

where $\varepsilon$ is an aerodynamic calibration parameter. Results presented in Refs. 5, 6, and 7 suggest that this parameter is a measure of the flow compression which occurs at the measurement matrix and is a function of Mach number and angle of attack. The complete aerodynamic model may be written as

$C_{p}(\theta)=\varepsilon+(1-\varepsilon) \cos ^{2}(\theta)=\cos ^{2}(\theta)+\varepsilon \sin ^{2}(\theta)$

Applying the definition of the pressure coefficient, the model may be written in terms of pressure as

$$
P\left(\theta_{i}\right)=q_{\infty}\left[\cos ^{2}\left(\theta_{i}\right)+\varepsilon \sin ^{2}\left(\theta_{i}\right)\right]+p_{\infty}
$$

where

$$
\theta_{i}=\lambda_{i}+\alpha_{\text {eff }}
$$

and $\lambda_{i}$ is the port latitude angle. Since the wing is a lifting surface, the measured angle of attack will not be the free-stream value. Instead a local flow angle, which is influenced by induced upwash at the leading edge, will be sensed. Thus an upwash calibration parameter, $\delta \alpha$, must be evaluated and included in the model in addition to the FADS aerodynamic calibration coefficient $(\varepsilon)$.

If one analyzes the lift field at the leading edge by superimposing a vortex in a uniform flow field with the vortex centered at the wing center of pressure, then the induced upwash angle is given by Ref. 11 as

$$
\alpha=\tan ^{-1}\left[\frac{\Gamma}{4 \pi V_{\infty} R}\right]
$$


where $\Gamma$ is the strength of the circulation field and $R$ is the vortex radius, measured as the distance from the the wing center of pressure to the leading edge. From the Kutta-Joukowski Law,

$$
\Gamma=\frac{L^{\prime}}{\rho V_{\infty}}=\frac{c \rho V_{\infty}^{2}}{2} \frac{c_{\ell}}{\rho V_{\infty}}=\frac{c V_{\infty} c_{\ell}}{2}
$$

$c$ is the geometric chord of the wing. Thus

$$
\delta \alpha=\tan ^{-1}\left[\frac{c c_{\ell}}{R 8 \pi}\right]
$$

and based on the theoretical data for $c_{\ell}$ and $x_{c p}$ computed earlier (Fig. 2(c)), the predicted upwash data can be evaluated. Predicted upwash values will be compared against experimentally derived values in the Results and Discussion section.

\section{Solution Algorithm}

The aerodynamic model is nonlinear and cannot be directly inverted to give airdata as a function of the measured pressures. Instead the measurements must be used to indirectly infer the airdata state using a nonlinear least-squares regression. Within each solution frame, the algorithm is linearized about a starting airdata value for each port location and the perturbations between the measured data and the model predictions are evaluated. This overdetermined system of perturbation equations is solved using weighted least squares. The resulting perturbation is added to the starting value and the system is re-linearized about the resulting update. The iteration cycle is repeated until algorithm convergence is reached-typically in two to four cycles. A detailed discussion of the convergence criterion used for the FADS algorithm is presented in Ref. 8. Since the Choleski Factorization techniques used to perform the regression are fairly standard, ${ }^{12}$ the numerical methods used will not be presented here. A detailed description of the regression algorithm can be found in Refs. 5 and 8 .

\section{Results and Discussion}

Results of the wind-tunnel tests will be presented in this section. First, data from the aerodynamic calibrations will be presented, and the resulting upwash value will be compared against a theoretically computed value. Using the results of the calibration tests, the performance of the system will be quantitatively evaluated over a variety of conditions.

\section{Calibration}

The calibration parameters $\delta \alpha$ and the FADS aerodynamic calibration coefficient $(\epsilon)$, were estimated from the wind-tunnel data by substituting measured values of geometric angle of attack, static pressure, and dynamic pressure into the aerodynamic model and comparing the model's pressure predictions to the pressures actually measured. Residuals between the measured and predicted pressures were used to infer the values of the calibration parameters using nonlinear regression. The resulting calibration data are presented in Figs. 5(a) and (b). The data bear a strong qualitative resemblance to calibration results extracted from the F-18 HI-FADS flight and wind-tunnel data (Refs. 5 and 9 ).

A comparison of the predicted upwash and experimentally derived upwash calibration values is presented in Fig. 5(a). Plotted on the ordinate axis is $\delta \alpha$, plotted on the abscissa is the true (geometric) angle of attack. Four data curves are presented, the theoretically derived curve, and the measured values at $35 \mathrm{kn}, 45 \mathrm{kn}$, and at maximum dynamic pressure. The upwash curve is symmetric as a function of angle of attack, this is as expected since the wing section is symmetrical. Tunnel airspeed did not exhibit any significant influence on the angle-of-attack calibration, and since upwash is primarily a function of the total lift coefficient, this is also as expected.

Tunnel blockage, however, exhibited a considerable influence. In the low-angle-of-attack region between $\pm 5^{\circ}$ where the tunnel blockage is less than 10 percent, the comparisons between the experimental and theoretical upwash curves are excellent. However, at higher angles of attack, the blockage effects significantly alter the flow characteristics of the lift field. Referring to Fig. 5(a), the theoretical upwash curve is linear between $\alpha= \pm 10^{\circ}$, and does not significantly break until $\alpha= \pm 30^{\circ}$. The experimental upwash curve is linear in the region between $\alpha= \pm 10^{\circ}$, and breaks significantly beyond $\alpha= \pm 20^{\circ}$. Clearly, blockage effects cause the wing to separate sooner than would occur in the absence of tunnel blockage. While the upwash data are probably not quantitatively valid (for a free-flying wing), the repeatability of the data suggest that the pressure matrix does indeed sense the true local angle of attack at the wing leading edge.

Calibration data for the FADS aerodynamic calibration coefficient are presented in Fig. 5(b). The FADS aerodynamic calibration coefficient is plotted on the ordinate axis, and the geometric angle of attack is plotted on the abscissa. As with the upwash curve, the aerodynamic calibration curve is also symmetric. Tunnel airspeed exhibited a minor effect on the FADS aerodynamic calibration coefficient, which grows as a function of airspeed, especially at low angles of attack. The curves consistently exhibit an inflection point between $\alpha= \pm 15^{\circ}$ and $\pm 20^{\circ}$. This inflection is almost certainly caused by the leeward surface of the wing becoming separated at higher angles of attack. The minor 
inflection point that occurs near $\alpha=0^{\circ}$ is probably a minor effect resulting from tunnel blockage. At $\alpha=0^{\circ}$, the tunnel is essentially unblocked, and the compression effect caused by blockage is quite small. At higher angles of attack, the compression effect is caused by angle-of-attack effects and tunnel blockage (which is also a function of angle of attack). Thus the level of the curves jumps slightly. Again, while the data for the FADS aerodynamic calibration coefficient are probably not quantitatively valid (for a free-flying wing), the repeatability of the data suggest that the pressure matrix does indeed sense the compression which is occurring at the wing leading edge.

\section{System Performance}

Systematic trends in the calibration parameters were extracted by curve fitting and interpolated to generate a series of tabular breakpoints, which were hardcoded into the estimation algorithm. Utilizing calibration data from the tests at maximum dynamic pressure, the system performance was evaluated for a set of known angle-of-attack conditions. Based on timeaveraged pressure distribution for the leading edge, the FADS algorithm was used to estimate values for geometric angle of attack, dynamic pressure, and tunnel static pressure. The estimated dynamic pressure and static pressure values were subsequently summed to compute total pressure. Recall that for wind-tunnel testing ambient pressure should equal the total pressure in the tunnel outside of the boundary layer.

Figure 6 presents a sample model-to-data comparison for maximum dymamic pressure and $\alpha=0^{\circ}$. The surface pressure is plotted on the ordinate axis and the surface incidence angle is plotted on the abcissa. Notice that the data compare very well with the predicted model values, indicating an excellent model "fit." Figure 7(a) shows a comparison of the FADS angle-of-attack estimate with the measured value. Figure 7 (b) presents a similar comparison for ambient (total) pressure. The standard deviation between the computed and actual angle-of-attack curves is approximately $0.25^{\circ}$, and the standard deviation between the estimated and measured ambient pressure curves is approximately $0.8 \mathrm{lb} / \mathrm{ft}^{2}$.

The results of these tests indicate that the FADS algorithms developed for nose-based systems are directly applicable to wing leading-edge locations. To apply the algorithms to the wing leading edge, only the aerodynamic calibration data tables need to be changed. The significance of this result is that the algorithms developed for the RT-FADS system, with their inherent real-time and fault-tolerant characteristics, need not be reformulated.

\section{Concluding Remarks}

A flush airdata sensing (FADS) system was installed on the leading edge of an unswept symmetrical airfoil. A series of low-speed wind-tunnel tests were performed to evaluate the feasibility of locating a FADS matrix on a wing leading edge. As a result of the tests it is concluded that the aerodynamic models formulated for use on an aircraft nosetip are directly applicable to a wing leading edge and that the calibration process is much the same for a wing leading edge as for a nosetip. Furthermore, the agreement between the airdata estimates of the FADS algorithm and the measurements in the wind tunnel suggest that such wing-based airdata systems can be calibrated to a similarly high degree of accuracy.

The results of these tests indicate that the FADS algorithms developed for nose-based systems are directly applicable to wing leading-edge locations. To apply the algorithms to the wing leading edge, only the aerodynamic calibration data tables need to be changed. The significance of this result is that the algorithm developed for the real-time (RT)-FADS system, with its inherent real-time and extensively developed faulttolerant characteristics, need not be reformulated. To completely validate the leading-edge (LE)-FADS concept, further tests must be performed using swept-wing configurations, nonsymmetrical airfoils, sharper leading edges, and dynamic conditions.

\section{References}

${ }^{1}$ Gracey, William, Measurement of Aircraft Speed and Altitude, NASA-RP 1046, 1980.

${ }^{2}$ Siemers, Paul M., III, Wolf, H., and Henry, M.W., "Shuttle Entry Air Data System (SEADS)-Flight Verification of an Advanced Air Data System Concept," AIAA-88-2104, 1988.

${ }^{3}$ Larson, Terry J., and Siemers, Paul M., III, Use of Nose Cap and Fuselage Pressure Orifices for Determination of Air Data for Space Shuttle Orbiter Below Supersonic Speeds, NASA TP-1643, 1980.

${ }^{4}$ Larson, Terry J., Whitmore, Stephen A., Ehernberger, L.J., Johnson, J. Blair, and Siemers, Paul M., III, Qualitative Evaluation of a Flush Air Data System at Transonic Speeds and High Angles of Attack, NASA TP-12716, 1987.

${ }^{5}$ Whitmore, Stephen A., Moes, Timothy R., and Larson, Terry J., "High Angle-of-Attack Flush Airdata Sensing System," J. of Aircraft, vol. 29, no. 5, Sept.Oct. 1992, pp. 915-919. 
${ }^{6}$ Moes, Timothy R., and Whitmore, Stephen A., Preliminary Results From an Airdata Enhancement Algorithm With Application to High-Angle-of-Attack Flight, NASA TM-101737, 1991.

${ }^{7}$ Whitmore, Stephen A., and Moes, Timothy R., The Effects of Pressure Sensor Acoustics on Airdata Derived From a High-Angle-of-Attack Flush Airdata Sensing (HI-FADS) System, AIAA-91-0671, Jan. 1991. Also printed as NASA TM-101736, 1991.

${ }^{8}$ Whitmore, Stephen A., Moes, Timothy R., and Leondes, Cornelius, T., Failure Detection and Fault Management Techniques for a Pneumatic High-Angleof-Attack Flush Airdata Sensing (HI-FADS) System, AIAA-92-0263, Jan. 1992. Also printed as NASA TM-4335, 1992.
${ }^{9}$ Whitmore, Stephen A., Moes, Timothy R., and Leondes, Cornelius T., "Development of a Pneumatic High-Angle-of-Attach Flush Airdata Sensing System," vol. 52, Integrated Technology Methods and Applications in Aerospace Systems Design, Control and Dynamic Systems, Advances in Theory and Applications, Academic Press, San Diego, CA, 1992, pp. 453-511.

${ }^{10}$ PANDA-A Program for Analysis and Design of Airfoils, Desktop Aeronautics, Stanford, CA, 1988.

${ }^{11}$ Kuethe, Arnold M., and Chow, Chyuen-Yen, Foundations of Aerodynamics: Bases of Aerodynamic Design, John Wiley \& Sons, New York, NY, 1976, pp. 90-96.

${ }^{12}$ Golub, Gene, H., and Van Loan, Charles F., Matrix Computations, Johns Hopkins University Press, Baltimore, MD, 1983, pp. 86-90. 


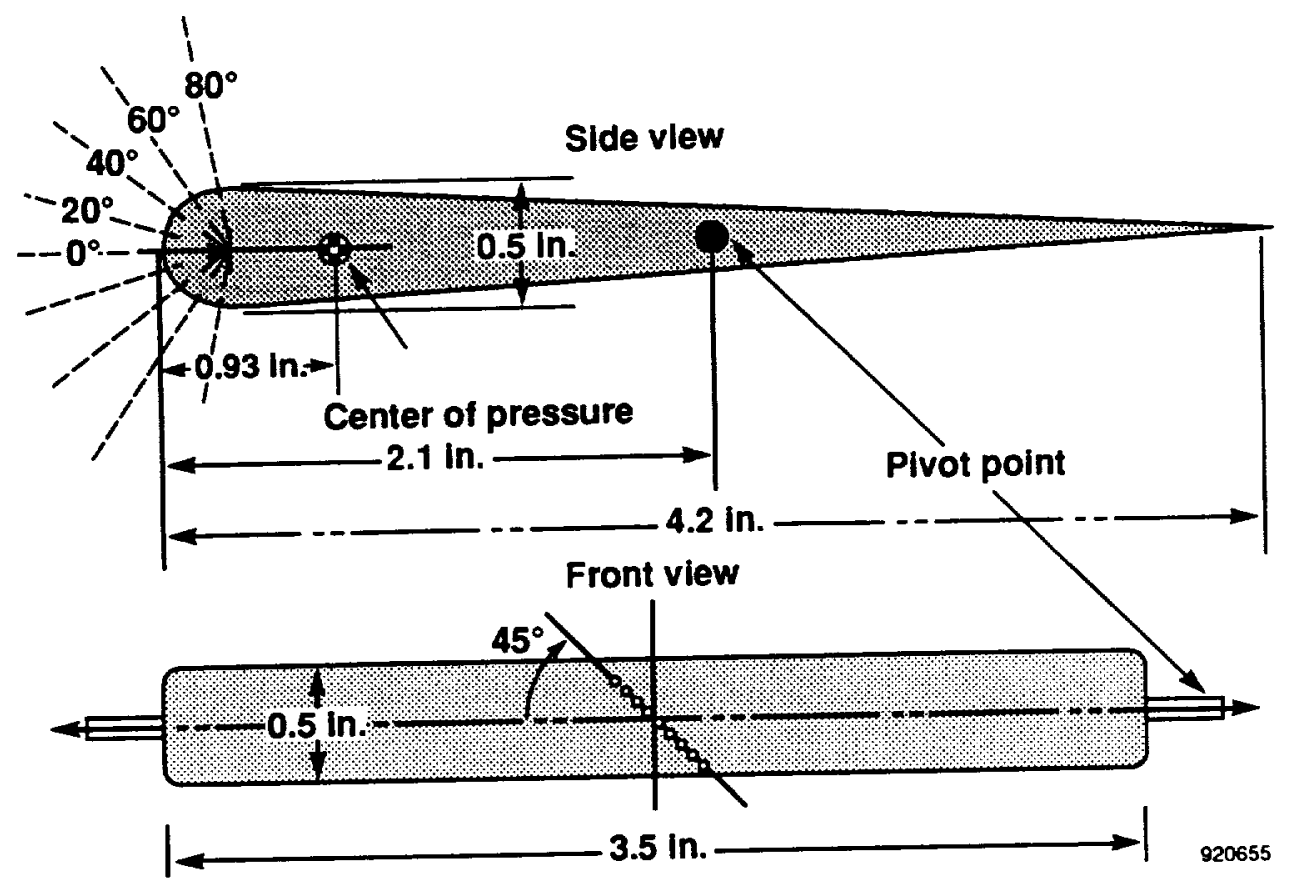

Fig. 1 Schematic of LE-FADS test wing.

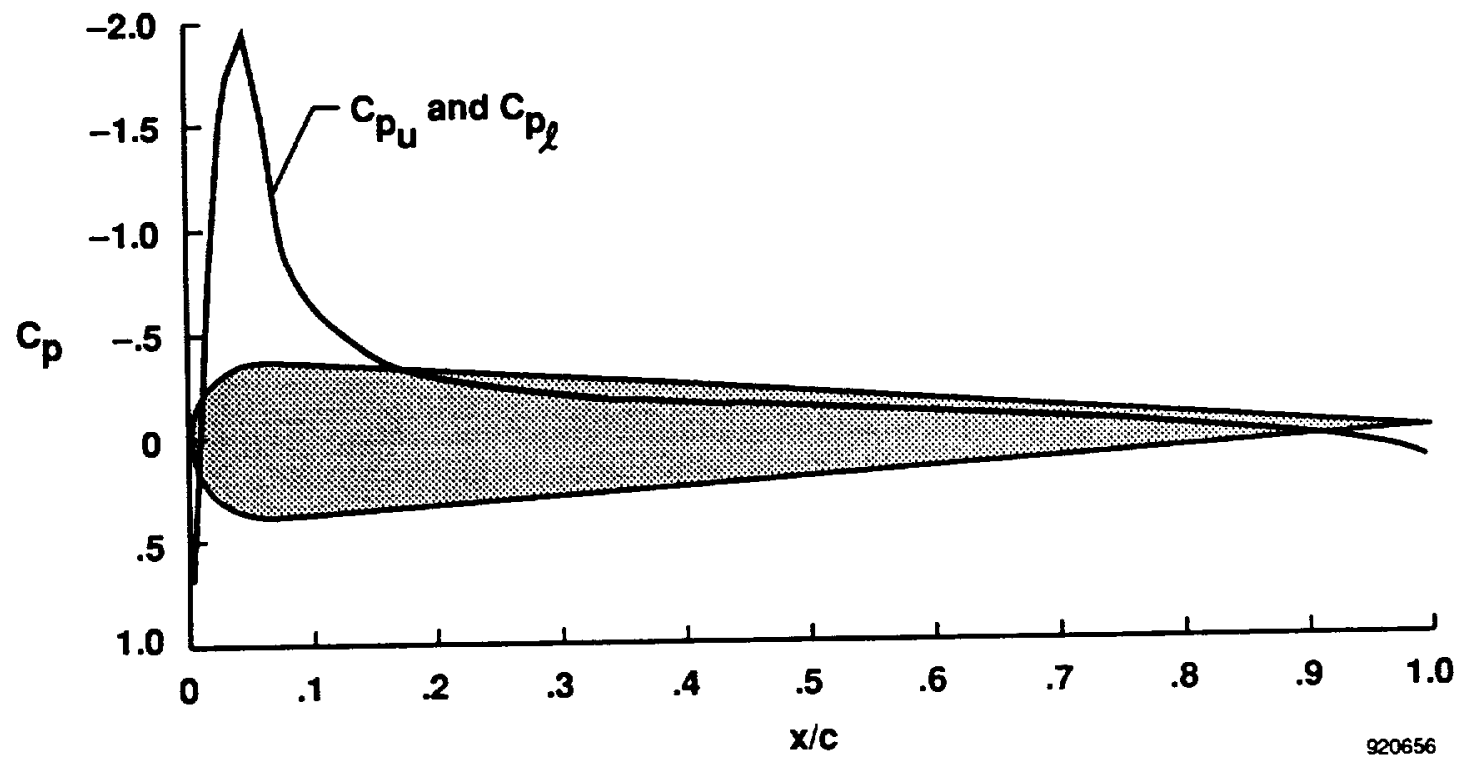

(a) Pressure distribution at $\alpha=0^{\circ}, M_{\infty}=0$.

Fig. 2 Theoretical LE-FADS wing characteristics. 


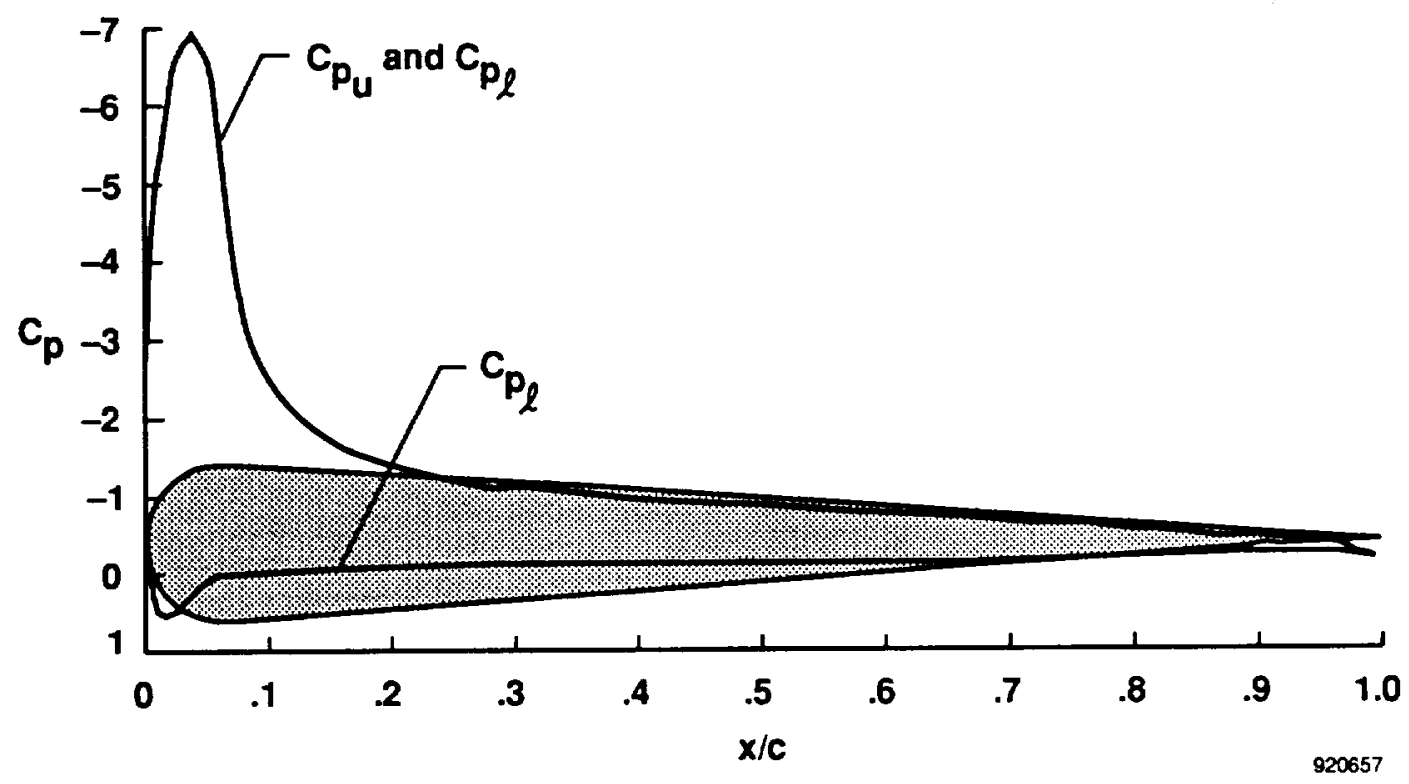

(b) Pressure distribution at $\alpha=10^{\circ}, M_{\infty}=0$.

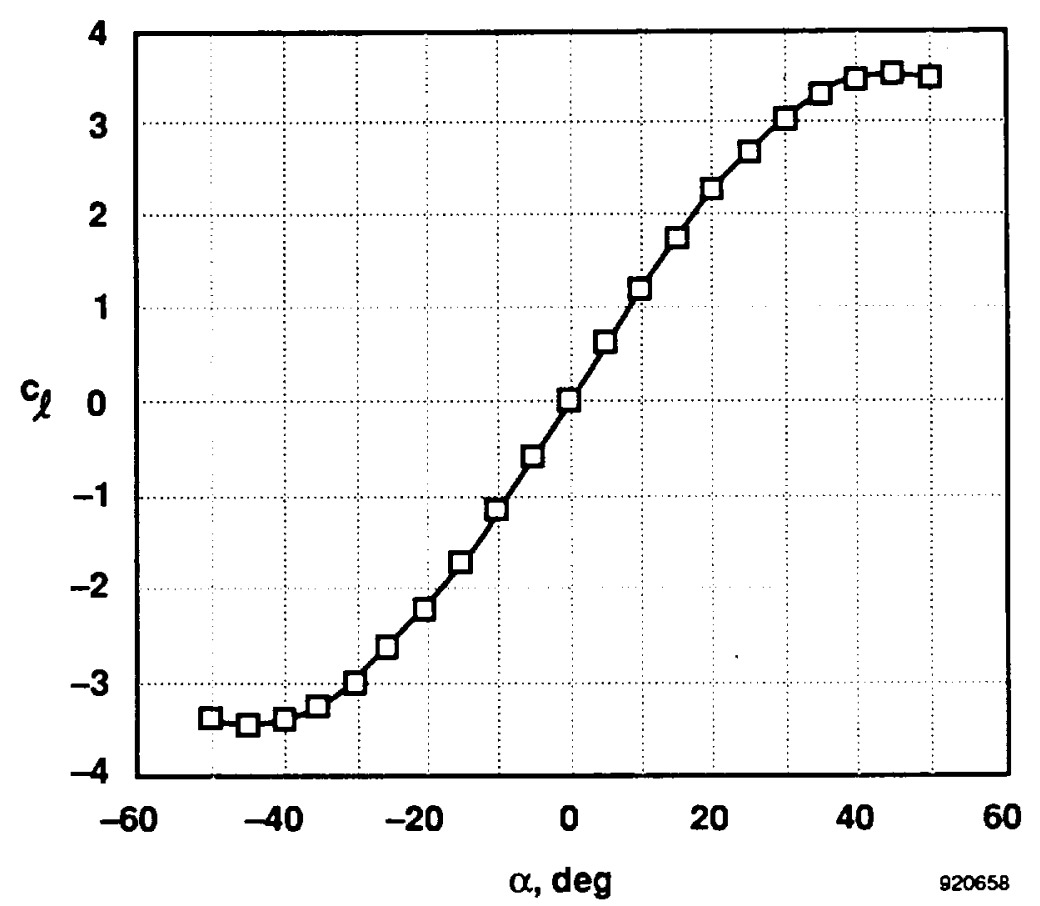

(c) Sectional lift coefficient.

Fig. 2 Concluded. 




Fig. 3 Schematic of experimental setup. 


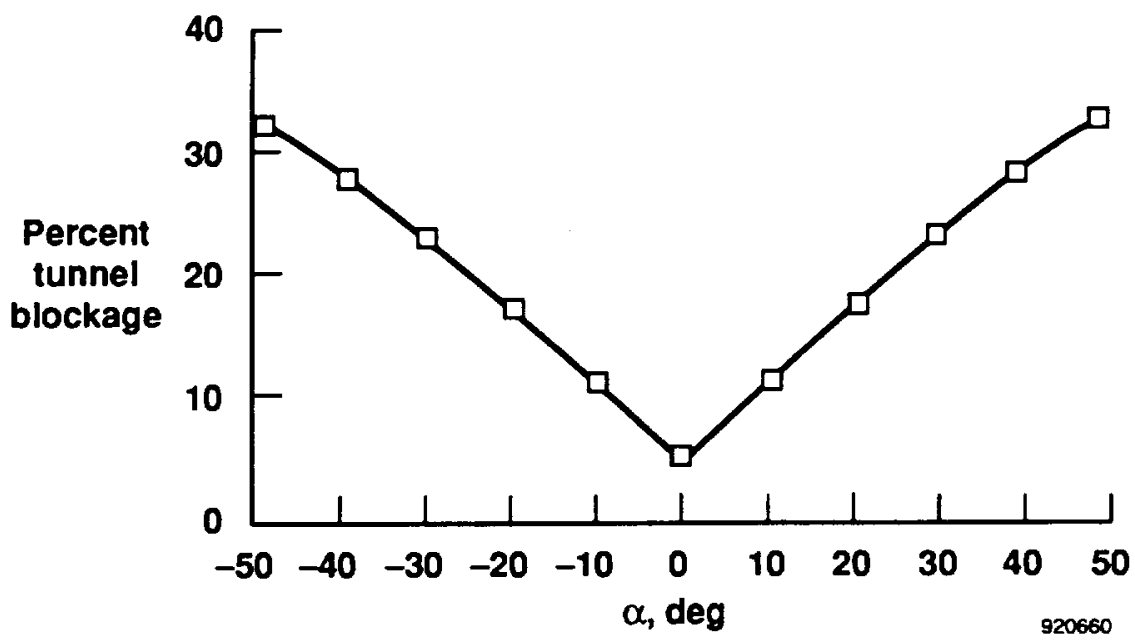

(a) Blockage as a function of angle of attack.

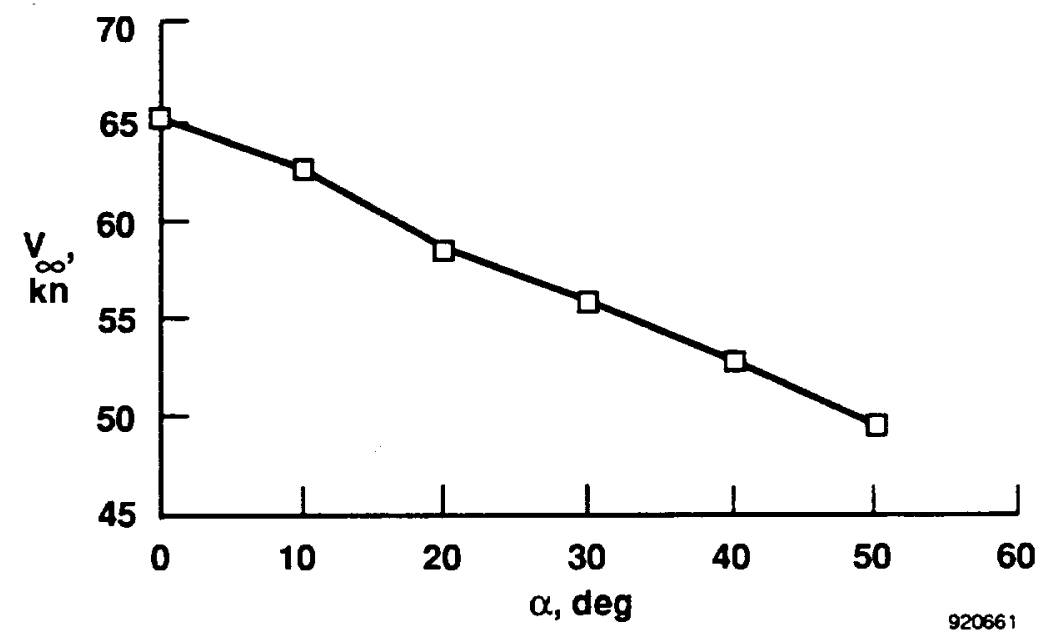

(b) Blockage effects on airspeed.

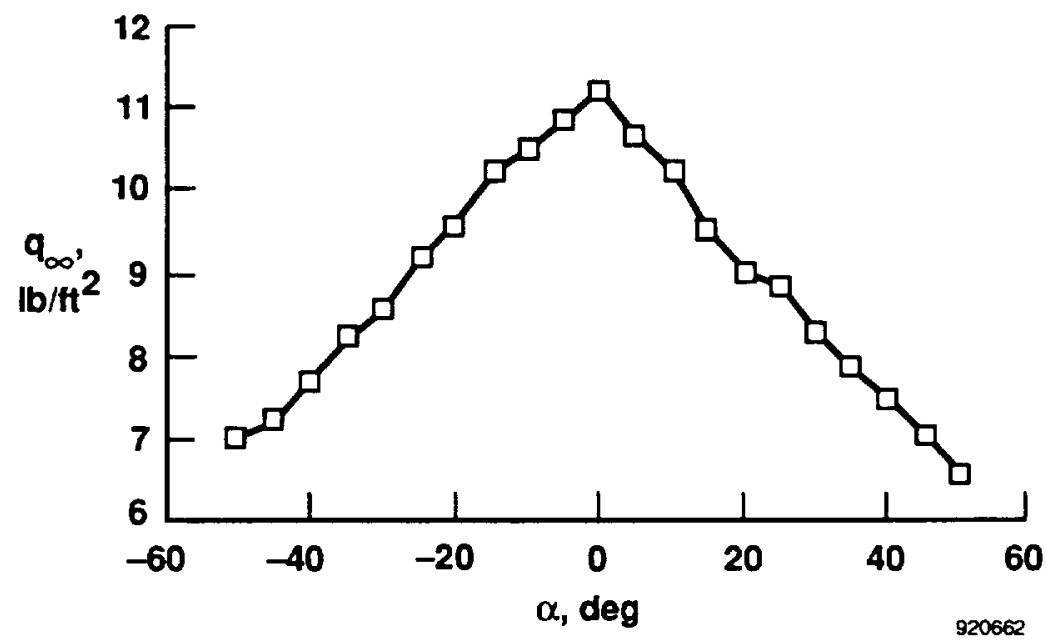

(c) Blockage effects on dynamic pressure.

Fig. 4 Effects of wind-tunnel blockage $(1750 \mathrm{r} / \mathrm{min})$. 

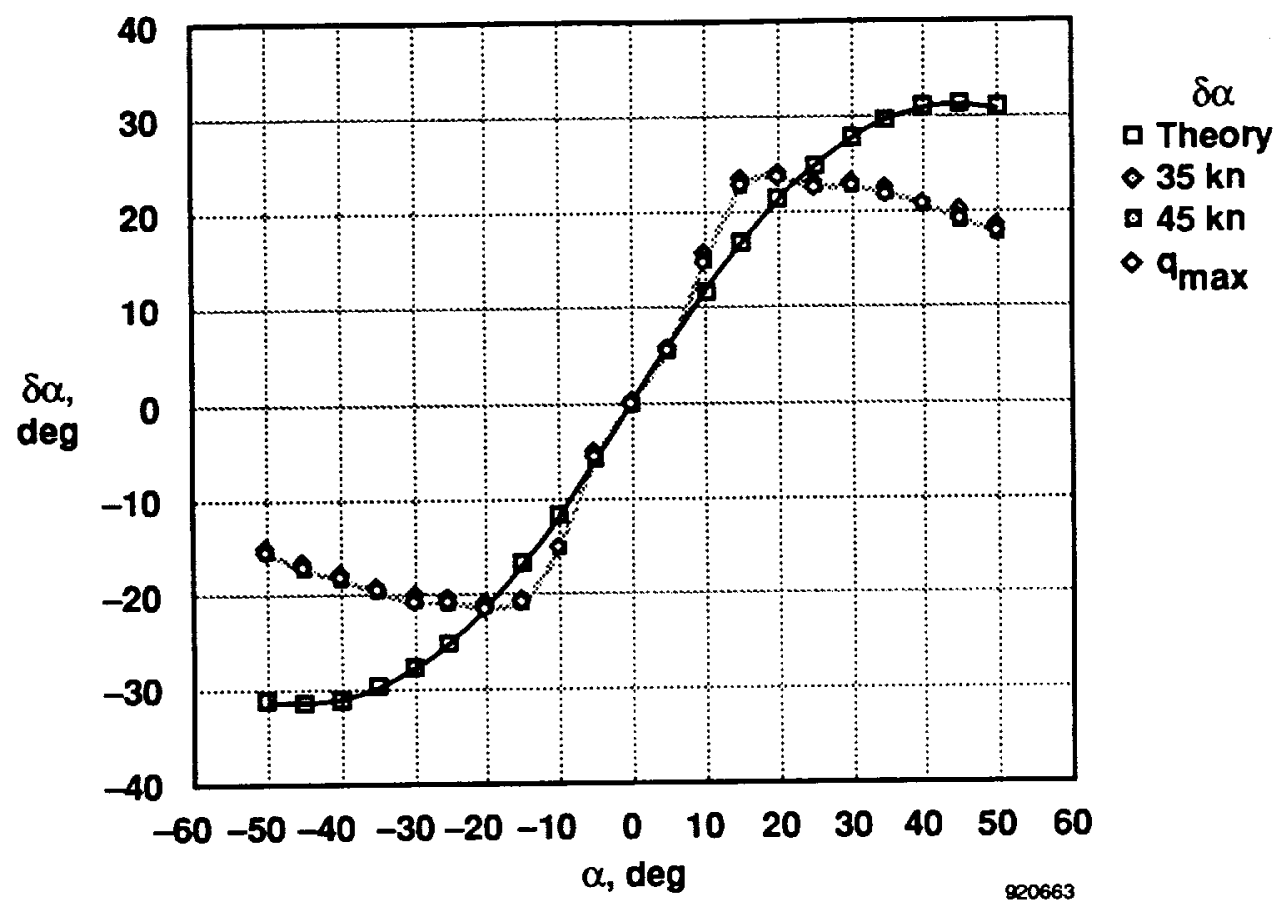

(a) Upwash calibration.
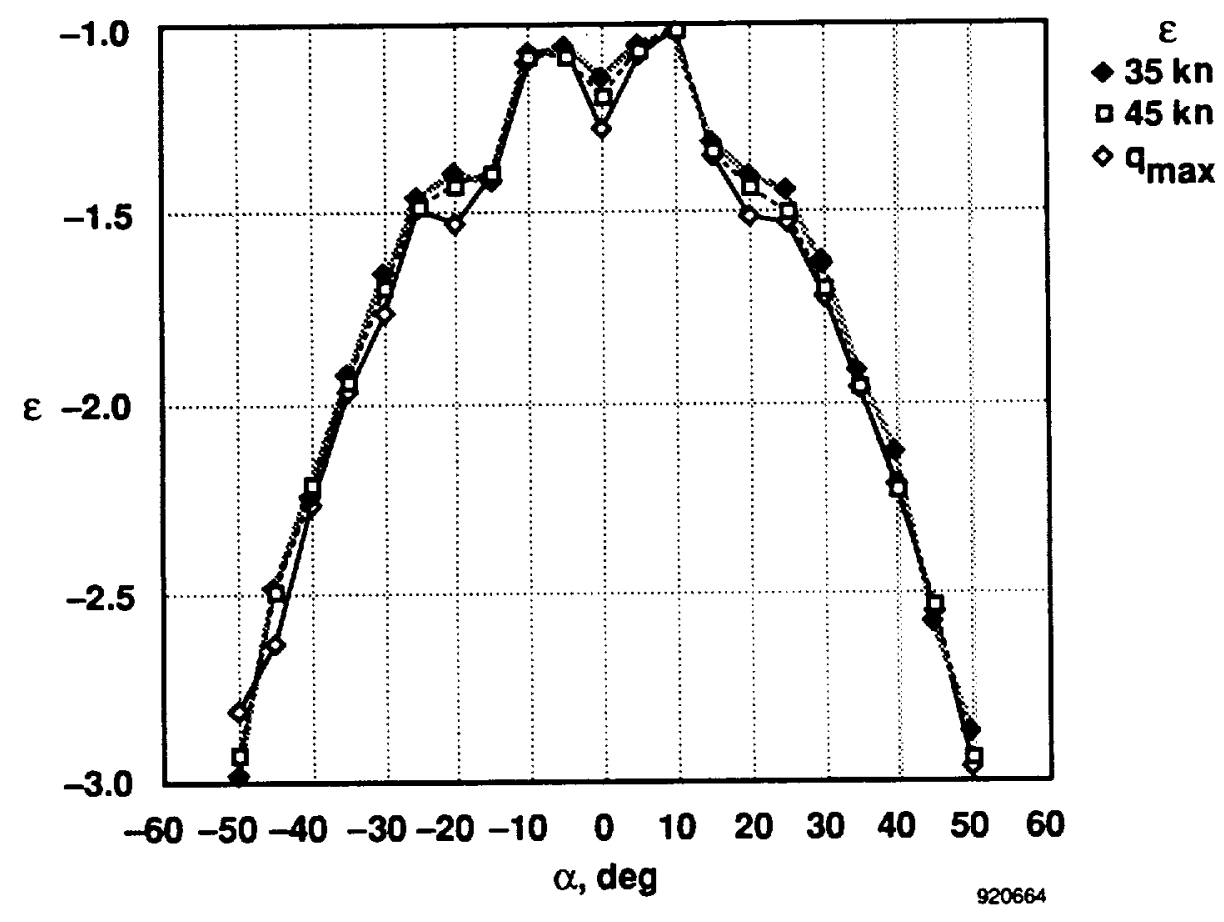

(b) Aerodynamic calibration.

Fig. 5 FADS calibration results. 


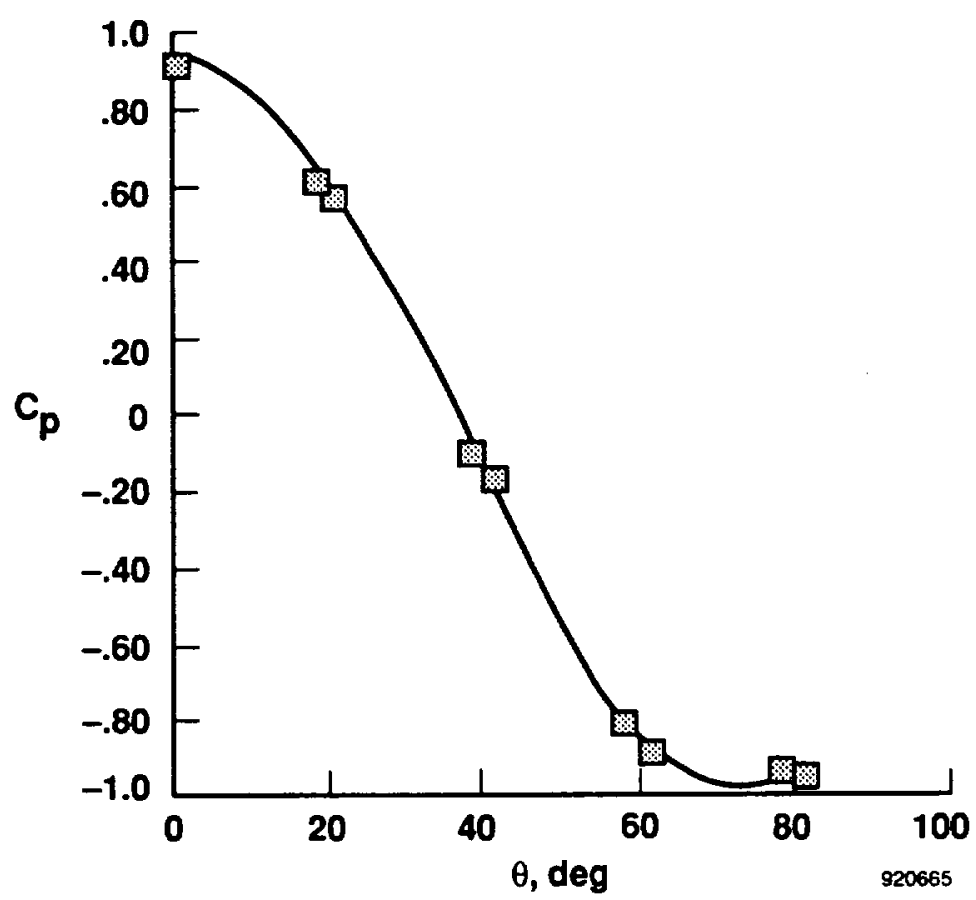

Fig. 6 Comparison of LE-FADS pressure data to algorithm fit. 


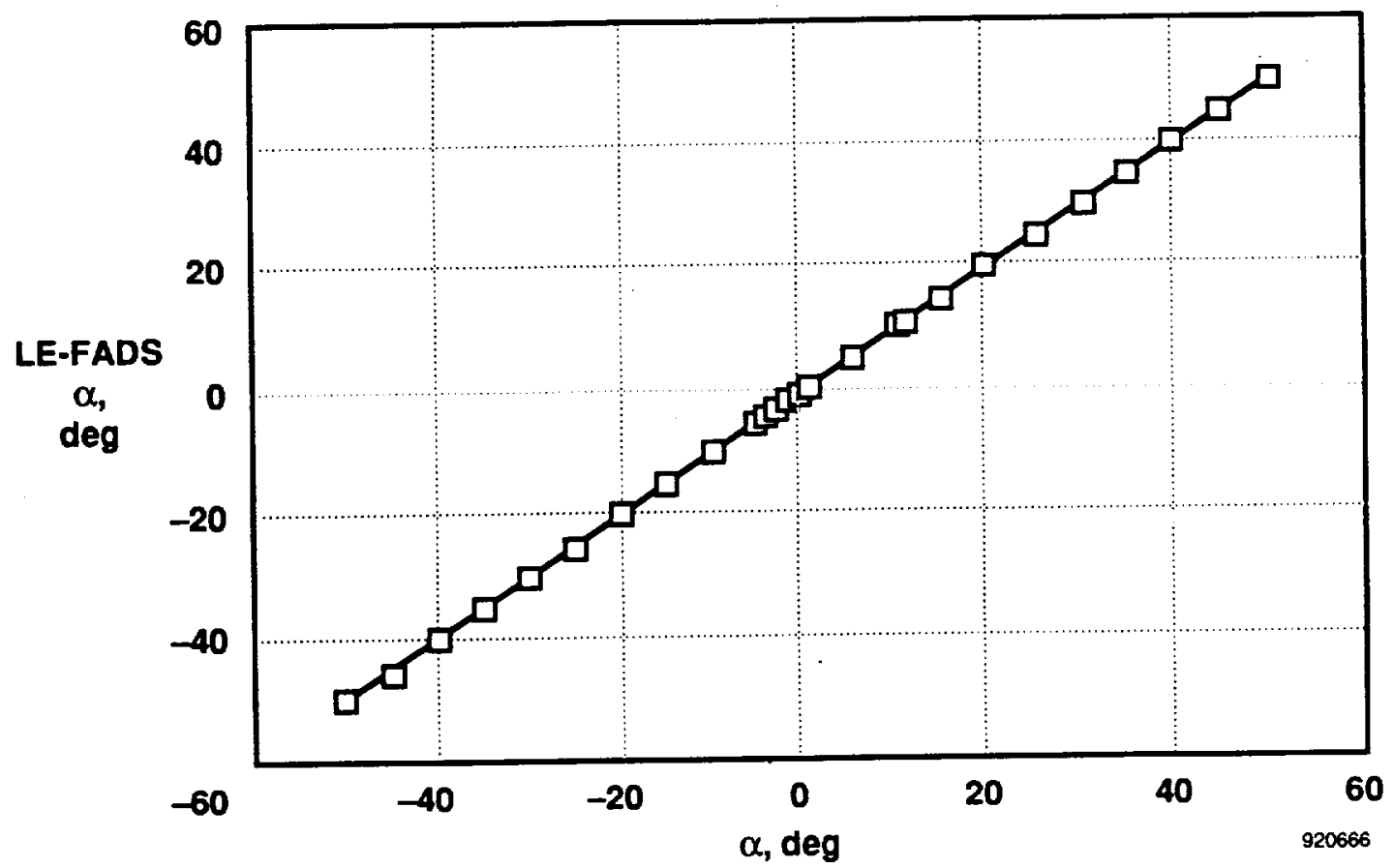

(a) Angle of attack.

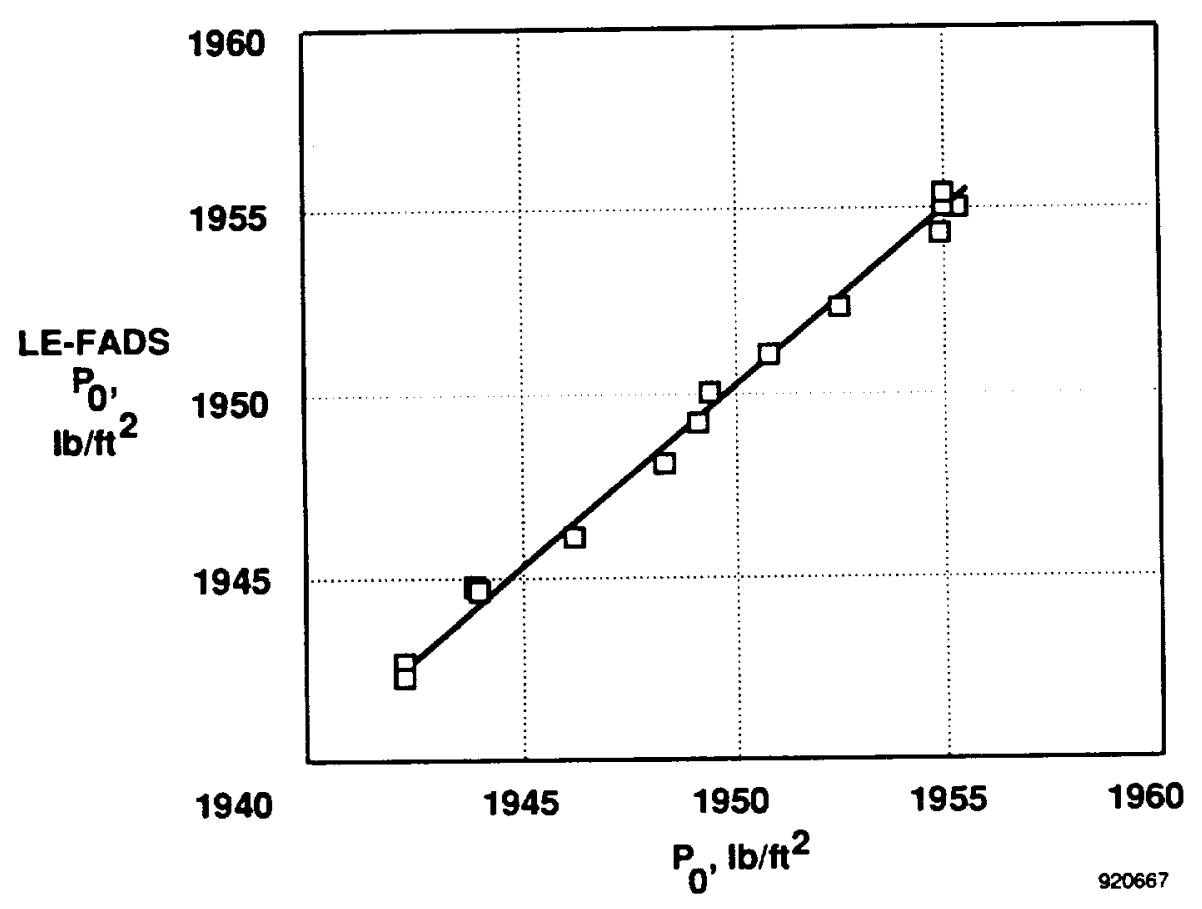

(b) Stagnation pressure.

Fig. 7 FADS performance evaluation. 


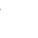




\section{REPORT DOCUMENTATION PAGE}

Public reponing burden tar this collection of iniormation is estimated to average 1 hour por response. including the time for roviowing instructions, searching existing dath cources.

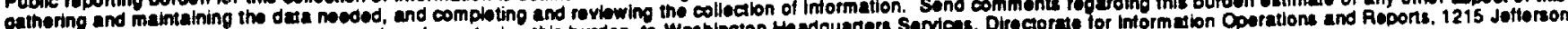

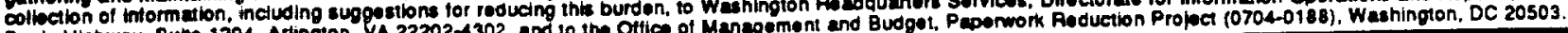

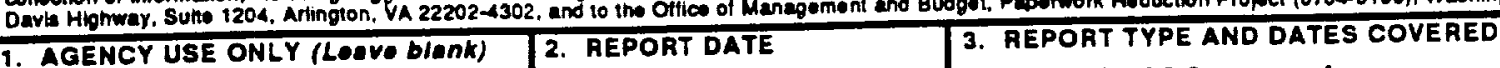

1. AGENCY USE ONLY (Leave biank)

February 1993

Technical Memorandum

4. TITLE AND SUBTITLE

Application of a Flush Airdata Sensing System to a Wing Leading Edge

(LE-FADS)

6. AUTHOR(S)

Stephen A. Whitmore, Timothy R. Moes, Mark W. Czemiejewski, and Douglas A. Nichols

7. PERForming organization name(S) AND ADDRESS(ES)

8. PERFORMING ORGANIZATION

P.O. Box 273

Edwards, CA 93523-0273

9. SPONSORING/MONITORING AGENCY NAME(S) AND ADDRESS(ES)

National Aeronautics and Space Administration

Washington, DC 20546-0001 REPORT NUMBER

H-1886

WU 505-68-40

10. SPONSORING/MONITOAINC AGENCY REPORT NUMBER

NASA TM-104267

\section{SUPPLEMENTARY NOTES}

Prepared as AIAA-93-0634 for the AIAA 31st Aerospace Sciences Meeting, Reno, Nevada, January 11-14, 1993.

12a. DISTRIBUTION/AVAILABILITY STATEMENT

12b. DISTRIBUTION CODE

Unclassified - Unlimited

Subject Category 06

13. AgSTRACT (Maximum 200 words)

This paper investigates the feasibility of locating a flush airdata sensing (FADS) system on a wing leading edge where the operation of the avionics or fire control radar system will not be hindered. The leading-edge FADS system (LE-FADS) was installed on an unswept symmetrical airfoil and a series of low-speed wind-tunnel tests were conducted to evaluate the performance of the system. As a result of the tests it is concluded that the aerodynamic models formulated for use on aircraft nosetips are directly applicable to wing leading edges and that the calibration process is similar. Furthermore, the agreement between the airdata calculations for angle of attack and total pressure from the LE-FADS and known wind-runnel values suggest that wing-based flush airdata systems can be calibrated to a high degree of accuracy. Static wind-tunnel tests for angles of attack from $-50^{\circ}$ to $50^{\circ}$ and dynamic pressures from 3.6 to $11.4 \mathrm{lb} / \mathrm{ft}^{2}$ were performed.

14. SUBJECT TERMS

Airspeed; Angle of attack; Flush airdata; Noninstrusive airdata; Wing leading edge

15. NUMBER OF PAGES

17

16. PRICE CODE

$\mathrm{AO3}$

\begin{tabular}{|l|l|l}
\hline 17. SECURITY CLASSIFICATION & $\begin{array}{l}\text { 18. SECURITY CLASSIFICATION } \\
\text { OF REPORT } \\
\text { OF THIS PAGE } \\
\text { Unclassified }\end{array}$ & $\begin{array}{l}\text { 19. SECURITY CLLASSIFICATION } \\
\text { OF ABSTRACT }\end{array}$ \\
\hline
\end{tabular}

